\title{
The middle-term result of laparoscopic sleeve gastrectomy in Chinese obesity patients in a single hospital, with the review of literatures and strategy for gastric stenosis
}

\author{
Jianzhong Di ${ }^{1,2}$, Chen Wang ${ }^{1}$, Pin Zhang ${ }^{1}$, Xiaodong Han ${ }^{1}$, Weijie Liu ${ }^{1}$, Hongwei Zhang ${ }^{1}$ \\ ${ }^{1}$ Department of General Surgery, the Sixth People's Hospital Affiliated to Shanghai Jiao Tong University, Shanghai 200233, China; ${ }^{2}$ Department of \\ General Surgery, Shigatse People's Hospital, Shigatse 857000, China \\ Contributions: (I) Conception and design: J Di, H Zhang; (II) Administrative support: P Zhang; (III) Provision of study materials or patients: P Zhang, \\ X Han, W Liu; (IV) Collection and assembly of data: J Di, C Wang; (V) Data analysis and interpretation: J Di, C Wang, H Zhang; (VI) Manuscript \\ writing: All authors; (VII) Final approval of manuscript: All authors. \\ Correspondence to: Dr. Hongwei Zhang. Department of Bariatric and Metabolic Surgery, Shanghai Jiao Tong University Affiliated Sixth People's \\ Hospital, Shanghai 200233, China. Email: zhw00sub@163.com.
}

\begin{abstract}
Background: The aim of this study was to evaluate the medium-term metabolic results of laparoscopic sleeve gastrectomy (LSG) in Chinese patients.

Methods: We performed a retrospective study of our LSG results between February 2011 and February 2015, and conducted a literature review of stenosis after LSG during a recent 10-year period.

Results: Of 142 patients in our study, the mean initial body mass index (BMI) was $38.34 \mathrm{~kg} / \mathrm{m}^{2}$, the mean age of the patients (44 males and 98 females) was 29.36 years. All patients were followed up for at least 36 months. There was a significant reduction in BMI and blood lipid levels. For one case with gastric stenosis $(0.7 \%)$, resolution was achieved by conversion to gastric bypass, with a satisfied result in weight loss and diabetes remission. The incidence of stenosis after LSG in previous studies was $0.1-3.5 \%$. Among the cases of stenosis, $83.0 \%$ resolved with dilation, and the remainder resolved with a surgical procedure.

Conclusions: LSG is a relatively safe surgical option for weight loss as a primary procedure in our study. Stenosis after LSG requires diagnosis and treatment promptly. Surgery can be a feasible treatment option, but care must be taken to avoid complications.
\end{abstract}

Keywords: Sleeve gastrectomy; stenosis; stricture; type 2 diabetes mellitus (T2DM); obesity

Submitted Sep 20, 2018. Accepted for publication Dec 04, 2018.

doi: 10.21037/atm.2018.12.28

View this article at: http://dx.doi.org/10.21037/atm.2018.12.28

\section{Introduction}

Bariatric and metabolic surgery for morbid obesity and obesity with comorbidity has proved effective and is becoming popular throughout the world (1-3). Laparoscopic sleeve gastrectomy (LSG) is one type of bariatric and metabolic surgery with lower morbidity and complication rates and similar effectiveness when compared with laparoscopic Roux-en-Y gastric bypass or laparoscopic biliopancreatic diversion with duodenal switch $(1,4)$. The number of LSGs being performed worldwide has sharply increased in recent years (5). Furthermore, the early results of LSG have been so promising, it is now under investigation as a solitary surgical procedure for weight loss and obesity related diseases $(6,7)$.

In Chinese populations, there was little research of bariatric and metabolic surgery in treating obesity with middle or large term result. The objective of this study was to report on the preliminary middle-term results of LSG in Chinese patients. 
Table 1 Surgical results of LSG

\begin{tabular}{|c|c|c|c|c|c|}
\hline Parameter & Pre-op & 3rd-Mo & 6th-Mo & 12th-Mo & 36th-Mo \\
\hline Weight (kg) & $106.85 \pm 17.69$ & $91.60 \pm 19.46$ & $90.38 \pm 18.72$ & $82.25 \pm 19.14^{\mathrm{a}}$ & $68.13 \pm 17.56^{a}$ \\
\hline BMI $\left(\mathrm{kg} / \mathrm{m}^{2}\right)$ & $38.34 \pm 5.69$ & $32.20 \pm 4.92^{\mathrm{a}}$ & $29.42 \pm 3.80^{\mathrm{a}}$ & $26.71 \pm 3.57^{\mathrm{a}}$ & $25.66 \pm 6.52^{\mathrm{a}}$ \\
\hline Hipline (cm) & $121.96 \pm 8.65$ & $112.5 \pm 6.48^{a}$ & $103.4 \pm 9.07^{\mathrm{a}}$ & $102.75 \pm 9.39^{a}$ & $99.75 \pm 10.78^{a}$ \\
\hline Cholesterol (mmol/L) & $5.11 \pm 0.86$ & $5.08 \pm 0.87$ & $4.56 \pm 0.65$ & $5.20 \pm 0.54$ & $4.45 \pm 0.16^{\mathrm{a}}$ \\
\hline $\mathrm{HDL}(\mathrm{mmol} / \mathrm{L})$ & $1.09 \pm 0.20$ & $1.00 \pm 0.17$ & $0.97 \pm 0.11$ & $1.24 \pm 0.04^{\mathrm{a}}$ & $1.21 \pm 0.18$ \\
\hline $\mathrm{LDL}(\mathrm{mmol} / \mathrm{L})$ & $3.39 \pm 0.91$ & $3.27 \pm 0.87$ & $2.94 \pm 0.46$ & $3.36 \pm 0.50$ & $2.74 \pm 0.17^{\mathrm{a}}$ \\
\hline
\end{tabular}

a, $\mathrm{P}<0.05$ vs. perioperative values. Ideal body weight index $=25 \mathrm{~kg} / \mathrm{m}^{2} ; \% \mathrm{EWL}$ values were not analyzed. LSG, laparoscopic sleeve gastrectomy; Pre-op, preoperative; Mo, postoperative month; EWL, excess weight loss; BMI, body mass index; HDL, high-density lipoprotein; LDL, low-density lipoprotein.

\section{Methods}

Between February 2011 and February 2015, a total of 142 patients underwent LSG. The human research review board of our institution approved the study and all patients provided written informed consent.

Following the latest guidelines for metabolic surgery from the Chinese Society for Metabolic and Bariatric Surgery, the inclusion criteria were as follows: Patients with a body mass index (BMI) over $35 \mathrm{~kg} / \mathrm{m}^{2}$ and associated comorbidities or a BMI over $30 \mathrm{~kg} / \mathrm{m}^{2}$ but with severe comorbidities, such as osteoarticular problems, diabetes, or dyslipidemia, were included in the study. Patients with established diagnoses of type 1 diabetes, latent autoimmune diabetes in adulthood, malignancy, debilitating disease, unresolved psychiatric illness or substance abuse were excluded.

Data recorded included patient demographics, serum lipid levels, and surgical complications. The means and standard deviations were computed for continuous variables and evaluated using independent $t$ tests. All statistics were two-tailed and were calculated with SPSS statistical software (version 20.0; SPSS Inc., Chicago, IL, USA). Values of $\mathrm{P}<0.05$ were considered statistically significant.

The incidence of stenosis after LSG during a recent 10-year period was examined. A literature search of English-language studies was conducted using the following terms: sleeve, sleeve gastrectomy, complications, stenosis, and strictures.

\section{Surgical procedure}

Prophylactic heparin and antibiotics were administered preoperatively. After the induction of general anesthesia, LSG was performed using 4 ports, a carbon dioxide pneumoperitoneum was achieved to $15 \mathrm{mmHg}$ pressure, and all trocars were placed under direct vision. During the procedure, the assistant introduces, via the mouth, a 37-French bougie inside the gastric lumen to prevent narrowing of the tubular stomach. The short gastric vessels were taken down with the Harmonic scalpel (Ethicon Endosurgery) $5 \mathrm{~cm}$ proximal to the pylorus up to $1 \mathrm{~cm}$ from the angle of His using 4.8- $\mathrm{mm}$ green staples and 3.5-mm blue staples (Ethicon Echelon 60, EC60 stapler). This leaves a small gastric tubular pouch of $60-$ to $80-\mathrm{mL}$ capacity. The greater curvature, including the posterior fundus, is mobilized, and a methylene blue test is performed to test for intraoperative leaks. Bleeding at the staple line is controlled with metal clips. A $16 \mathrm{~F}$ round drain was placed in the sub-hepatic space along the suture line. All patients recovered in an intermediate care unit. The liquids were initiated on the fifth postoperative day and then the drain was removed.

\section{Results}

A total of 142 patients underwent LSG successfully without requiring conversion to the open technique. The mean age of the patients (44 males and 98 females) was 29.36 years. Detailed pre- and postoperative demographics are listed in Table 1. All patients were followed up for at least 36 months after surgery. The body weight, BMI, waistline and hipline were significantly reduced compared with baseline continuously. Mean BMI achieved within $28 \mathrm{~kg} / \mathrm{m}^{2}$ in 12 months after surgery. The serum lipid levels were 


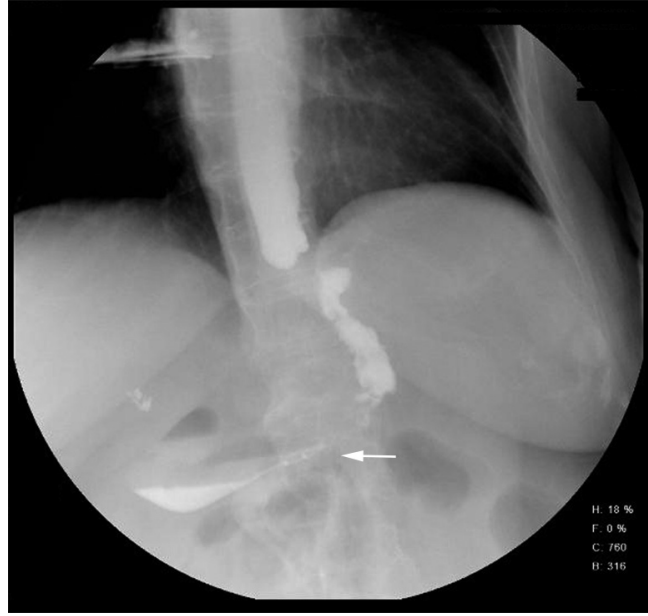

Figure 1 Upper abdominal gastrointestinal series shows a narrow passage in the middle sleeve at the 6th week after laparoscopic sleeve gastrectomy, indicated by the arrow.

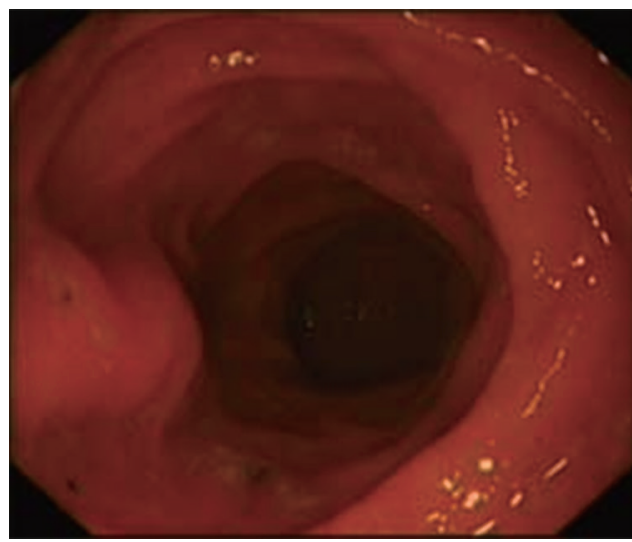

Figure 2 Gastroscopy shows no obvious stenosis.

significantly reduced. No mortality, leak, bleeding, or infection occurred. No severe malnutrition or vitamin or mineral deficiencies were observed in 36-month-follow-up.

However, one patient developed persistent stenosis $(1 / 142,0.7 \%)$. The operation was uneventful, and the patient was discharged after 1 week without any complaints. Two month postoperatively, patient reported postprandial nausea, vomiting and gastroesophageal reflux after drinking. An upper GI revealed a narrow passage at the middle sleeve (Figure 1) with delayed gastric emptying, but no obvious stenosis was observed (Figure 2). Several dilatations with a 45-F to 54-F bougie with supporting in nasojejunal enteral feeding were subsequently attempted without success

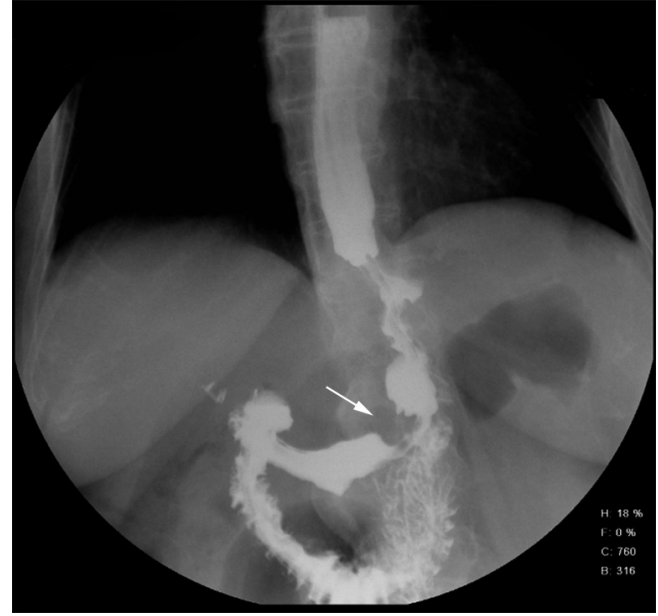

Figure 3 Upper abdominal gastrointestinal series shows the same narrow passage at the $3 \mathrm{rd}$ month after laparoscopic sleeve gastrectomy, indicated by the arrow.

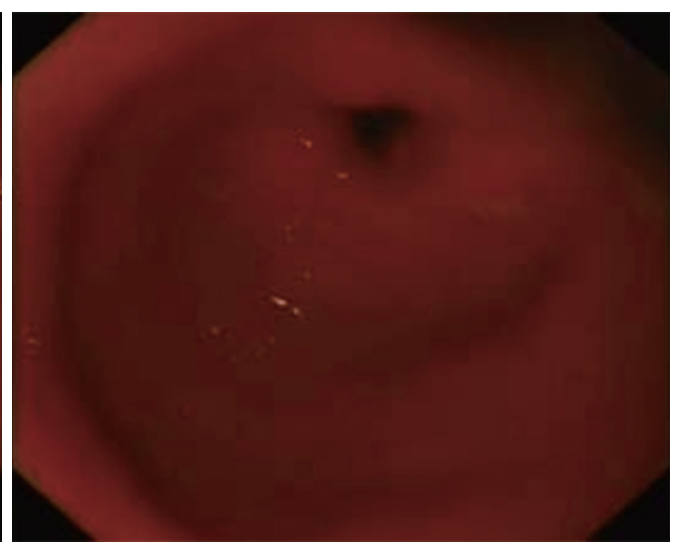

(Figure 3). A gastric bypass was performed finally. The middle sleeve adhered to the ventral abdominal wall (Figure 4) resulting in stenosis has been found during surgery, which caused twisting of the gastric lumen. Once the adhesion was loosened, the stenosis was relieved and the twist was corrected (Figure 5). The patient has been completely asymptomatic since the gastric bypass surgery (Figure 6). The assessment of nutritive status for the stenosis case was showed in Table 2.

The incidence of stenosis after LSG in recent ten years has been estimated at $0.1-3.5 \%$ (8-15) (Table 3). In 47 cases of gastric stenosis, $83.0 \%$ (39 cases) were relieved by dilation, one or several times, with different size bougies, 


\section{Page 4 of 8}

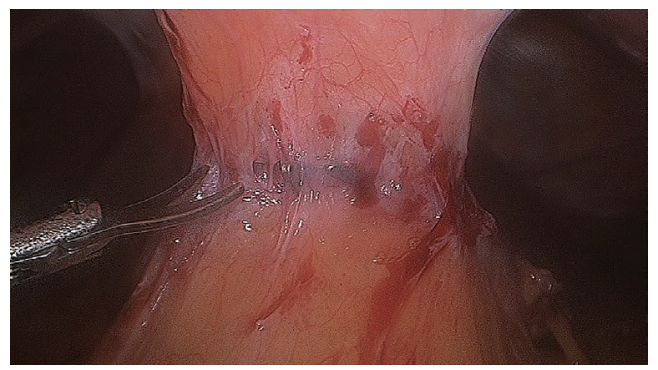

Figure 4 Upper sleeve shows adhesion to the ventral abdominal wall.

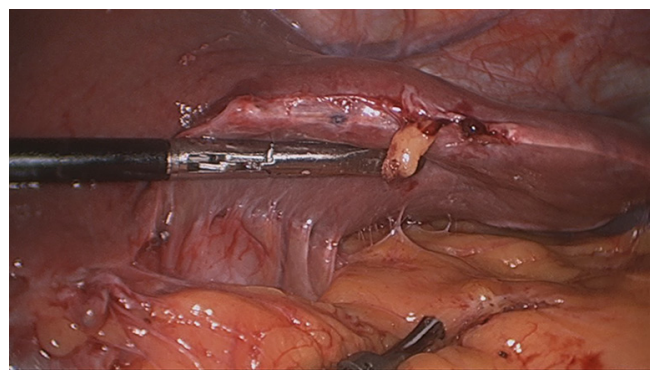

Figure 5 Final view of lysis of adhesions of the ventral abdominal wall.

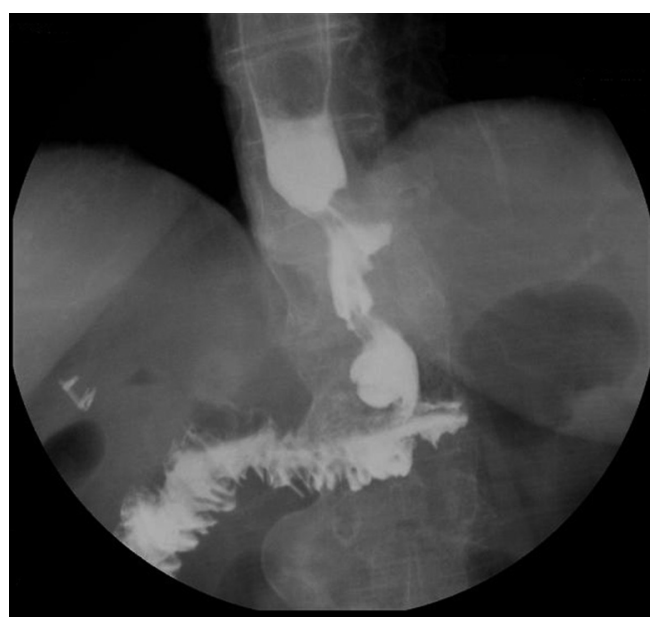

Figure 6 Upper abdominal gastrointestinal series shows resolution of stenosis after gastric bypass.

and only 4 cases were converted to gastric bypass, while 2 cases underwent resection of the stenotic segment, and 2 cases with conservative treatment. Seventeen cases (36.2\%) of stenosis occurred in the middle of the gastric lumen, whereas 15 occurred in the proximal third, and 15 occurred in the distal third (Table 4). However, despite the Levy's research, remained studies showed the most
Di et al. Middle-term result of sleeve gastrectomy in Chinese patients

stenosis occurred in the middle of the gastric lumen $(15 / 21$, $71.4 \%)$. No gastric bypasses were performed for treatment of stenosis in the proximal third of the lumen.

\section{Discussion}

Sleeve gastrectomy was first introduced by Marceau and Hess for the treatment of morbid obesity in the 1990s $(16,17)$. Since then, metabolic and bariatric surgery has become one of the most commonly performed types of gastrointestinal surgery for the treatment of type 2 diabetes mellitus (T2DM) with obesity worldwide. During the past few years, many encouraging reports have suggested that LSG is an effective, stand-alone procedure for T2DM with obesity (18). In this study, we report major perisurgical adverse outcomes in a recent cohort of patients undergoing the most common bariatric and metabolic surgical procedures performed by experienced surgeons in a single center in China. The surgical result confirms that LSG is a promising treatment for obesity in Chinese patients. All our patients had stabilization of weight within their normal range while avoiding the risk of malnutrition and being underweight. In addition, serum lipid levels were significantly decreased. Successful improvement of dyslipidemia can be translated into a decrease in mortality and further metabolic disorders. However, for only small number of patients who were diagnosed obesity with T2DM, this study didn't not contain the data or analysis of diabetes status.

No severe malnutrition or vitamin or mineral deficiencies were observed in 36-months follow up. Previous studies already showed the importance of pre- and post-operative assessment of the nutritional status of patients undergoing a bariatric intervention (19) and recommended adequate supplementation based on laboratory values. Regular follow-up and adequate multivitamin intake might have contributed to the resolution of the deficiency risk.

With the growing enthusiasm to perform LSG in morbidly obese patients, sleeve procedures composed $45.9 \%$ of the total metabolic surgeries in the world during 2014 (5). Although LSG has several advantages over other metabolic and bariatric procedures $(6,20-22)$, stenosis of the gastric sleeve is a complication of sleeve gastrectomy. As the number of LSG operations has increased sharply in recent years, so has the risk of postoperative stenosis.

Stenosis after LSG may result from multiple etiologies, including twisting of the gastric tube, indentation of the incisura within the gastric lumen, sharp angulation of 
Table 2 Assessment of nutritive status for the stenosis case

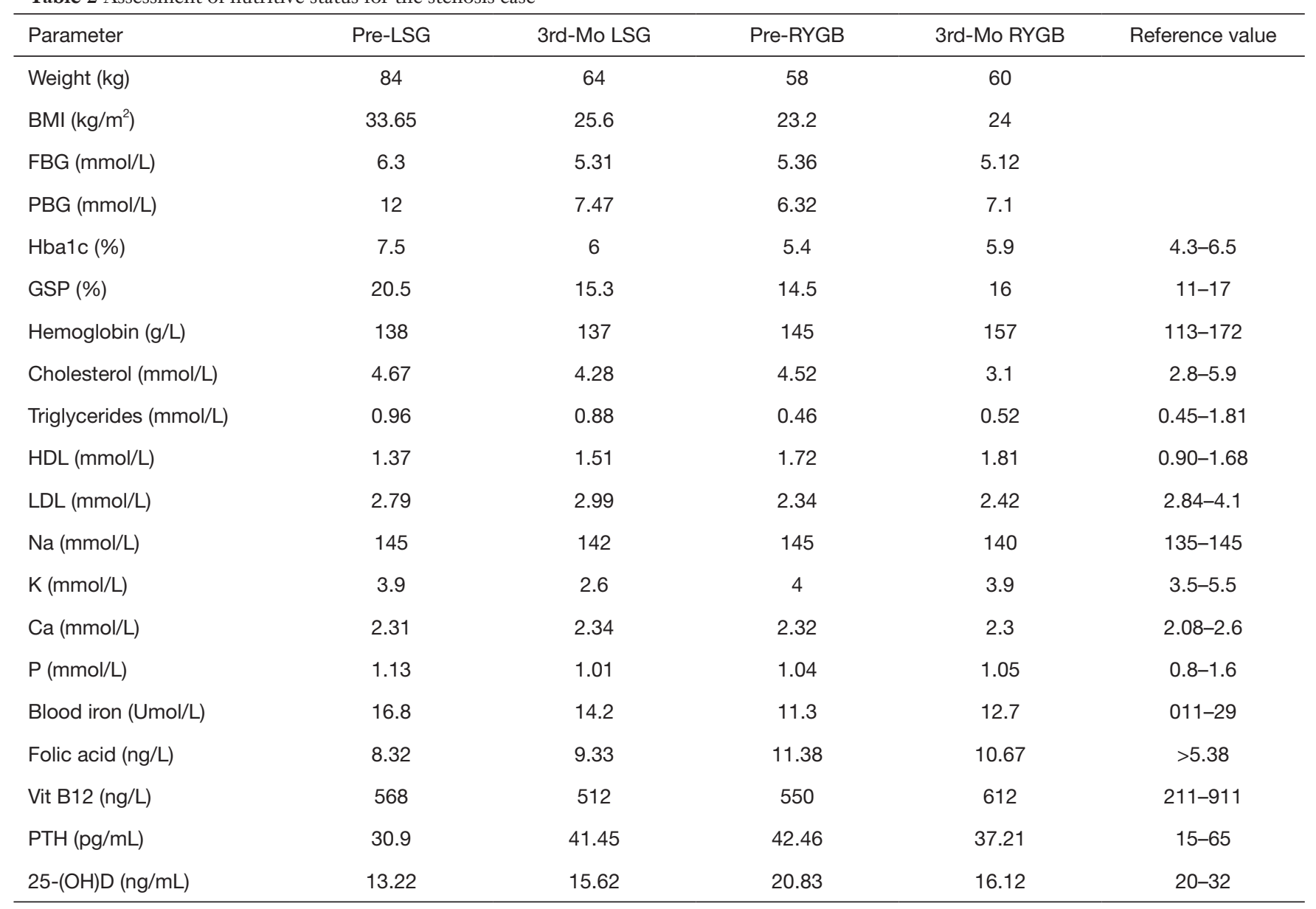

Pre-sleeve, pre-laparoscopic sleeve gastrectomy; 3rd-Mo LSG, 3rd postoperative month after laparoscopic sleeve gastrectomy; Pre-RYGB, pre-Roux-en-Y gastric bypass; 3rd-Mo RYGB, 3rd postoperative month after Roux-en-Y gastric bypass; BMI, body mass index; EWL, excess weight loss; FBG, fasting blood glucose; PBG, postprandial blood glucose; Hba1c, hemoglobin A1c; GSP, glycosylated serum protein; HDL, high-density lipoprotein; LDL, low-density lipoprotein; Na, serum sodium; K, serum potassium; CA, serum calcium; P, serum phosphorus; Vit B12, vitamin B12; PTH, parathyroid hormone; 25-(OH)D, 25-hydroxy vitamin D.

Table 3 Incidence of gastric stenosis after LSG and treatment

\begin{tabular}{|c|c|c|c|c|c|c|c|}
\hline Year & Author & $\mathrm{N}$ & Stenosis & Rate (\%) & \multicolumn{2}{|c|}{ Treatment } & Conservative \\
\hline 2008 & Lalor et al. & 148 & 1 & 0.70 & 1 & 0 & 0 \\
\hline 2008 & Mui et al. & 49 & 1 & 2 & 1 & 0 & 0 \\
\hline 2012 & Lacy et al. & 294 & 3 & 1.02 & 1 & 2 & 0 \\
\hline 2012 & Parikh et al. & 230 & 8 & 3.50 & 8 & 0 & 0 \\
\hline 2013 & Burgons et al. & 717 & 5 & 0.69 & 4 & 1 & 0 \\
\hline 2015 & Ramya et al. & - & 2 & - & 1 & 1 & 0 \\
\hline 2017 & Levy et al. & - & 26 & - & 23 & 1 & 2 \\
\hline
\end{tabular}

LSG, laparoscopic sleeve gastrectomy. 
Table 4 Stenosis location of LSG

\begin{tabular}{|c|c|c|c|}
\hline Year & Author & Stenosis location & $\mathrm{N}$ \\
\hline 2008 & Mui et al. & Angular incisure & 1 \\
\hline \multirow[t]{2}{*}{2012} & Lacy et al. & GE junction & 1 \\
\hline & & Central & 2 \\
\hline \multirow[t]{2}{*}{2012} & Parikh et al. & GE junction & 1 \\
\hline & & Central & 7 \\
\hline \multirow[t]{2}{*}{2013} & Burgons et al. & GE junction & 1 \\
\hline & & Central & 4 \\
\hline \multirow{3}{*}{2017} & & Middle third & 2 \\
\hline & & Proximal third & 11 \\
\hline & & Distal + proximal third & 1 \\
\hline \multirow[t]{2}{*}{ Overview } & $N(46 / 47)$ & Proximal third/middle third/distal third & $15 / 17 / 15$ \\
\hline & Bypass N (total 4) & Proximal third/middle third/distal third & $0 / 3 / 1$ \\
\hline
\end{tabular}

Proximal third includes GE junction. Middle third includes central part. Distal third includes angular incisure and antrum. LSG, laparoscopic sleeve gastrectomy; GE, gastroesophageal.

the stapler during the surgery (23), small bougie size, hematomas, edema (8), or adhesions (24). In our patient, twisting of the upper gastric tube due to middle sleeve adhesion was the underlying cause. Table 4 shows the possible locations of stenosis in the gastric tube. In most research, most stenoses occur at the level of the middle sleeve, whereas stenoses at the upper or lower sleeve happen rarely.

Most stenoses are symptomatic in the first 6 weeks after surgery (25), and the diagnosis of stenosis after LSG is based on symptoms and endoscopic examination. However, while upper GI and endoscopy provide good visualization of the gastric tube, detection of external adhesions may require other imaging methods. In our patient, both upper GI and gastroscopy showed only delayed emptying, and no stenosis was seen. This made us hesitant to redo the procedure. If an examination had been able to provide an overview of the outside of the pouch, the correct diagnosis could have been made earlier. Virtual pouchography (VPG) has been reported to facilitate such diagnoses. Other options include 3-dimensional computed tomography and $\mathrm{X}$-ray irradiation.
When faced with postoperative stenosis, it is always difficult for surgeons to decide when to intervene and whether to choose conservative treatment or a surgical procedure. Although, conversion of sleeve gastrectomy to gastric bypass has been advocated, conservative treatments, including stent insertion and bougie dilatation, have been reported to relieve symptoms successfully. Among reported stenoses, 3 patients with middle sleeve stenosis and 1 with lower sleeve stenosis underwent gastric bypass. No upper sleeve (gastroesophageal junction) stenoses were treated surgically. Dr. Vilallonga et al. (26) have reported that persistent stenosis can be managed by seromyotomy or wedge resection of the stomach, including the stricture, or stricture resection. However, these procedures have high complication and reoperation rates and should be performed with the greatest caution.

In our patient, multiple dilatations had minimal effect due to the adhesion at the middle sleeve level. Therefore, we performed a gastric bypass for upper gastric stenosis. This case confirmed the principle: solve the cause, solve the problem. 
Important limitations of our study include the inadequate sample size and duration to detect differences in longterm efficacy and safety. Therefore, our results should be regarded as preliminary. Second, it only included patients from a single hospital. Finally, this study was retrospective and affected the conclusions. A randomized clinical trial is indicated before any firm conclusions can be made.

\section{Conclusions}

Despite the limitations in our study, we conclude that LSG represents an effective procedure to treat obesity patients in Chinese patients. Our patients achieved obesity remission and dyslipidemia disorder control and reduced their metabolic risk. Symptoms and endoscopy may suggest the presence of stenosis after LSG, surgical procedures, such as conversion to gastric bypass, are a second-line treatment option, but care should be taken to avoid complications.

\section{Acknowledgements}

None.

\section{Footnote}

Conflicts of Interest: The authors have no conflicts of interest to declare.

Ethical Statement: The ethics of study was approved by the Ethics Committee of Shanghai Sixth People's Hospital (No. 2016-123) and written informed consent was obtained from all patients.

\section{References}

1. Buchwald H, Avidor Y, Braunwald E, et al. Bariatric surgery: a systematic review and meta-analysis. JAMA 2004;292:1724-37.

2. Schauer PR, Kashyap SR, Wolski K, et al. Bariatric surgery versus intensive medical therapy in obese patients with diabetes. N Engl J Med 2012;366:1567-76.

3. Schauer PR, Bhatt DL, Kirwan JP, et al. Bariatric Surgery versus Intensive Medical Therapy for Diabetes - 5-Year Outcomes. N Engl J Med 2017;376:641-51.

4. Dicker D, Yahalom R, Comaneshter DS, et al. Long-Term Outcomes of Three Types of Bariatric Surgery on Obesity and Type 2 Diabetes Control and Remission. Obes Surg 2016;26:1814-20.
5. Angrisani L, Santonicola A, Iovino P, et al. Bariatric Surgery and Endoluminal Procedures: IFSO Worldwide Survey 2014. Obes Surg 2017;27:2290-92.

6. Young MT, Gebhart A, Phelan MJ, et al. Use and Outcomes of Laparoscopic Sleeve Gastrectomy vs Laparoscopic Gastric Bypass: Analysis of the American College of Surgeons NSQIP. J Am Coll Surg 2015;220:880-5.

7. Charalampakis V, Seretis C, Daskalakis M, et al. The effect of laparoscopic sleeve gastrectomy on quality of life: A prospective cohort study with 5-years follow-up. Surg Obes Relat Dis 2018;14:1652-8.

8. Burgos AM, Csendes A, Braghetto I. Gastric stenosis after laparoscopic sleeve gastrectomy in morbidly obese patients. Obes Surg 2013;23:1481-6.

9. Lalor PF, Tucker ON, Szomstein S, et al. Complications after laparoscopic sleeve gastrectomy. Surg Obes Relat Dis 2008;4:33-8.

10. Mui WL, Ng EK, Tsung BY, et al. Laparoscopic Sleeve Gastrectomy in Ethnic Obese Chinese. Obes Surg 2008;18:1571-4.

11. Lacy A, Ibarzabal A, Pando E, et al. Revisional surgery after sleeve gastrectomy. Surg Laparosc Endosc Percutan Tech 2010;20:351-6.

12. Boza C, Salinas J, Salgado N, et al. Laparoscopic sleeve gastrectomy as a stand-alone procedure for morbid obesity: report of 1,000 cases and 3-year follow-up. Obes Surg 2012;22:866-71.

13. Parikh A, Alley JB, Peterson RM, et al. Management options for symptomatic stenosis after laparoscopic vertical sleeve gastrectomy in the morbidly obese. Surg Endosc 2012;26:738-46.

14. Kalaiselvan R, Ammori BJ. Laparoscopic median gastrectomy for stenosis following sleeve gastrectomy. Surg Obes Relat Dis 2015;11:474-7.

15. Levy JL, Levine MS, Rubesin SE, et al. Stenosis of gastric sleeve after laparoscopic sleeve gastrectomy: clinical, radiographic and endoscopic findings. Br J Radiol 2018;91:20170702.

16. Hess DS, Hess DW. Biliopancreatic diversion with a duodenal switch. Obes Surg 1998;8:267-82.

17. Marceau P, Biron S, Bourque RA, et al. Biliopancreatic diversion with a new type of gastrectomy. Obes Surg 1993;3:29-35.

18. Brito JP, Montori VM, Davis AM. Metabolic Surgery in the Treatment Algorithm for Type 2 Diabetes: A Joint Statement by International Diabetes Organizations. JAMA 2017;317:635-6. 
19. Damms-Machado A, Friedrich A, Kramer KM, et al. Pre- and post- operative nutritional deficiencies in obese patients undergoing laparoscopic sleeve gastrectomy. Obes Surg 2012;22:881-9.

20. Gagner M, Gumbs AA, Milone L, et al. Laparoscopic sleeve gastrectomy for the super-super-obese (body mass index $>60 \mathrm{~kg} / \mathrm{m}(2))$. Surg Today 2008;38:399-403.

21. Rubin M, Yehoshua RT, Stein M, et al. Laparoscopic sleeve gastrectomy with minimal morbidity. Early results in 120 morbidly obese patients. Obes Surg 2008;18:1567-70.

22. Gagner M, Deitel M, Kalberer TL, et al. The Second International Consensus Summit for Sleeve Gastrectomy, March 19-21, 2009. Surg Obes Relat Dis 2009;5:476-85.

23. Tucker ON, Szomstein S, Rosenthal RJ. Indications for

Cite this article as: Di J, Wang C, Zhang P, Han X, Liu W, Zhang H. The middle-term result of laparoscopic sleeve gastrectomy in Chinese obesity patients in a single hospital, with the review of literatures and strategy for gastric stenosis. Ann Transl Med 2018;6(24):479. doi: 10.21037/atm.2018.12.28 sleeve gastrectomy as a primary procedure for weight loss in the morbidly obese. J Gastrointest Surg 2008;12:662-7.

24. Dapri G. Reinforcing the staple line during laparoscopic sleeve gastrectomy: prospective randomized clinical study comparing three different techniques. Obes Surg 2010;20:462-7.

25. Rosenthal RJ; International Sleeve Gastrectomy Expert Panel, Diaz AA, et al. International Sleeve Gastrectomy Expert Panel Consensus Statement: best practice guidelines based on experience of $>12,000$ cases. Surg Obes Relat Dis 2012;8:8-19.

26. Vilallonga R, Himpens J, van de Vrande S. Laparoscopic management of persistent strictures after laparoscopic sleeve gastrectomy. Obes Surg 2013;23:1655-61. 